\title{
26300 - KETAMINE AND THE INCIDENCE OF EMERGENCE AGITATION IN CHILDREN UNDERGOING DENTAL REPAIR.
}

\section{Ibrahim Abu-Shahwan MD, Children Hospital Of Eastern Ontario, Ottawa, ONTARIO, Canada}

Introduction: Inhalation general anesthesia is frequently required in children undergoing surgical or diagnostic procedures. Sevoflurane has gained popularity because of the rapidity of induction and emergence from anesthesia, hemodynamic stability, and minor airway side effects (1). In spite of the clear usefulness of sevoflurane, concerns about quality of the emergence from anesthesia remain. The terms "agitation", "delirium", "excitement" or "confusion" has all been used to describe post anesthetic behavior in children (2). Several variables have been implicated in the development of behavioral problems or changes in children following surgery and/or anesthesia including pain, unfamiliar environment, separation from parents, and drugs effects. Increased incidence of emergence agitation following sevoflurane general anesthesia has been documented in several studies (3). The aim of this study was to evaluate the effect of adding intravenous ketamine $0.25 \mathrm{mg} / \mathrm{kg}$ at the end of surgery, on the incidence of emergence agitation in children undergoing dental repair.

Methods: After ethics committee approval we evaluated the effect of adding intravenous ketamine in children, 4 to 7 years of age, ASA I-III, undergoing dental repair with no extraction. Premedication with tylenol $(30 \mathrm{mg} / \mathrm{kg})$ and midazolam $(0.5 \mathrm{mg} / \mathrm{kg})$ were given 30 minutes before surgery to all children. Anesthesia was induced and maintained with a combination of sevoflurane/nitrous/oxygen. Mivacurium $0.25 \mathrm{mg} / \mathrm{kg}$ was used to facilitate nasotracheal intubation. Intravenous ketorolac $(1 \mathrm{mg} / \mathrm{kg})$ was used before endotracheal intubation. Children were allowed to regain spontaneous ventilation. Children in Group I (ketamine group) were given $0.25 \mathrm{mg} / \mathrm{kg}$ of ketamine 10 minutes before the end of surgery. Children in Group II (placebo group) were given saline and served as a control group. Patients demographic data, emergence agitation, and other side effect were recorded.

Results: Sixty-three of the 66 enrolled children completed the study. There was 31 children in Group I. Emergence agitation was diagnosed in 6 children in the ketamine group (19.3\%) and in 13 children in the placebo group (34.3\%). In both groups emergence agitation was less than 15 minutes duration and was successfully managed with fentanyl or propofol. There were no episodes of hallucinations or bad dreams in the ketamine group.

Discussion: The addition of a small dose of ketamine at the end of sevoflurane general anesthesia was effective in decreasing the incidence and severity of emergence agitation in children undergoing dental repair.

References: 1- Paed Anaesth 1999; 9: 299-304.

2- Paed Anaesth 2002; 12: 308- 312 3- Paed Anaesth 2000; 10: 419-424. 